\title{
ISCOOL - Interpretação de Textos através de Imagens e Tecnologias Semânticas
}

\author{
Bernardo Pereira Nunes ${ }^{1,2,4}$, Clara Ishikawa ${ }^{2}$, \\ Giseli Rabello Lopes ${ }^{5}$, Gilda Helena B. de Campos ${ }^{2,3}$ \\ ${ }^{1}$ Departamento de Informática, PUC-Rio \\ Rio de Janeiro - RJ - Brasil \\ bnunesdinf.puc-rio.br \\ ${ }^{2}$ Coordenação Central de Educação a Distância, PUC-Rio \\ Rio de Janeiro - RJ - Brasil \\ \{bernardo, clara\}@ccead.puc-rio.br \\ ${ }^{3}$ Departamento de Educação, PUC-Rio \\ Rio de Janeiro - RJ - Brasil \\ \{gilda\}@ccead.puc-rio.br \\ ${ }^{4}$ Universidade Federal do Estado do Rio de Janeiro, UNIRIO \\ Rio de Janeiro - RJ - Brasil \\ ${ }^{5}$ Universidade Federal do Rio de Janeiro, UFRJ \\ Rio de Janeiro - RJ - Brasil \\ giseliedcc.ufrj.br
}

\begin{abstract}
Resumo. A capacidade de avaliar criticamente informações textuais relativas ao material social, político e outros desempenha um papel crucial nos processos de formação de opinião e de tomada de decisão. Ela pode afetar pontos de vista pessoais e até mesmo provocar uma mudança social. Pensado para auxiliar nesse contexto, o jogo ISCOOL fornece ao usuário uma experiência de análise crítica de textos e desafia-o a combinar vários recursos em favor de reforçar as suas capacidades de interpretação e análise crítica visando que sejam mais precisas e embasadas. O ISCOOL beneficia-se de vários conjuntos de dados de referência, que provêm ao usuário informações sobre pessoas, organizações e locais, bem como definições de palavras e fatos históricos que servem como base para facilitar a compreensão textual. Dessa forma, é oferecido um contexto mais amplo a um texto inicial em que as informações relacionadas a este podem ser acessadas, interpretadas e compreendidas. Além disso, a interação não apenas com a ferramenta, mas também com outras pessoas, é estimulada através da possibilidade dos usuários gravarem os resultados obtidos para serem posteriormente analisados e discutidos.
\end{abstract}

\section{Cenário de Uso}

Embora o jogo ISCOOL possa ser utilizado em diversos contextos educacionais, dois grupos em particular foram identificados como sendo susceptíveis a se beneficiarem: 
CBIE-LACLO 2015

Anais dos Workshops do IV Congresso Brasileiro de Informática na Educação (CBIE 2015)

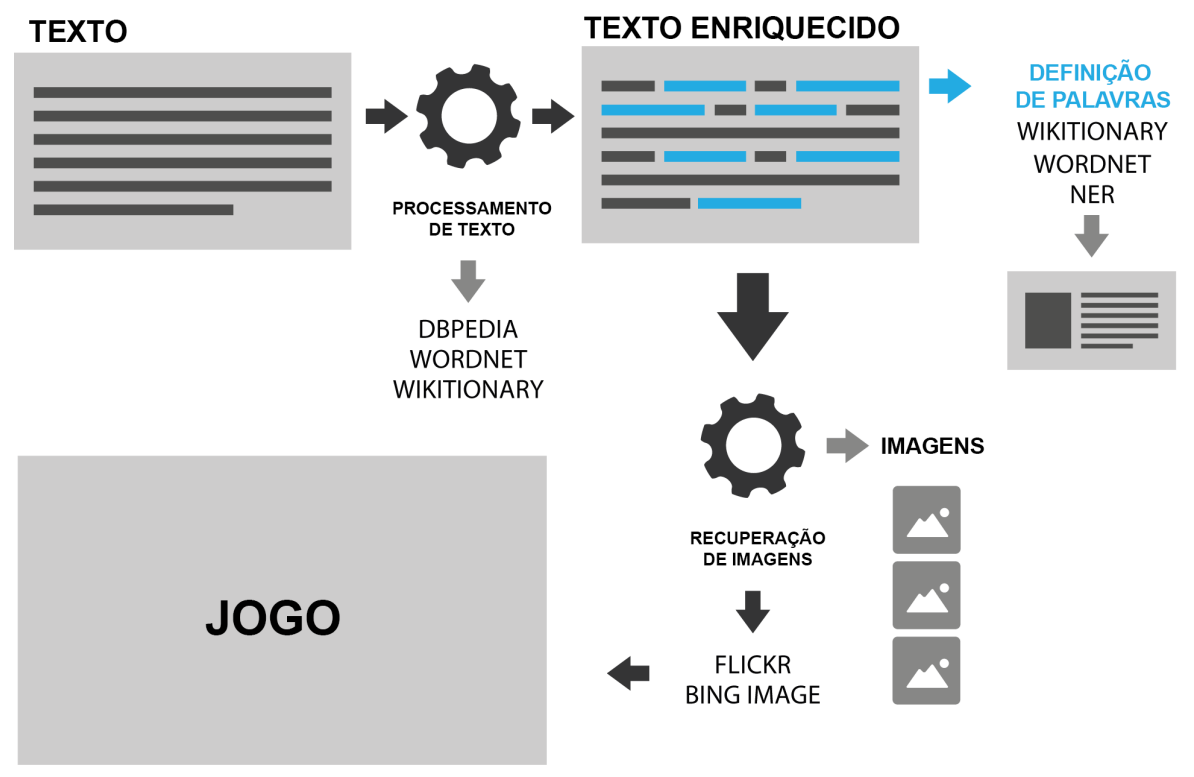

Figura 1. Workflow do ISCOOL.

- Pessoas com baixo nível de alfabetização. Um problema comum em países em desenvolvimento e muitas vezes ignorado pelos governos. As pessoas com baixos níveis de letramento podem ter dificuldades para ler e compreender textos longos e complexos. O ISCOOL permite o enriquecimento do vocabulário e interpretação de texto através de um jogo interativo em um ambiente amigável, porém competitivo que motiva o aluno a aprender e interagir com outros usuários.

- Pessoas com deficiência (deficiências auditivas, em particular). Pessoas que têm dificuldade de audição podem se sentir isoladas devido a barreiras linguísticas e as dificuldades de comunicação inerentes a sua condição. Desta forma, a fim de se comunicar (por exemplo, através da escrita/leitura), estes devem adquirir o que é essencialmente uma segunda língua (sendo a primeira a língua de sinais). O ISCOOL usa imagens para ajudar alunos com deficiência no processo de aprendizagem: como imagens são mais acessíveis e mais familiares no contexto da vida cotidiana, o aprendizado de uma segunda língua através de imagens pode ser mais confortável e motivador.

A versatilidade do ISCOOL garante que ele pode ser utilizado em vários contextos educativos diferentes no ensino fundamental e médio, bem como para adultos com deficiência ou baixos níveis educacionais. Assim, o jogo pode ser usado para apoiar não apenas aqueles com deficiência ou instrução limitada, mas também na aprendizagem de uma língua secundária.

Adicionalmente, um vídeo motivando os cenários de uso do ISCOOL está disponível em http: / / ccead.puc-rio.br/iscool/about.php.

\section{Desenvolvimento}

O jogo ISCOOL foi desenvolvido utilizando a linguagem de programação PHP e banco de dados MySQL. Além disso, diversas APIs, descritas ao longo desta seção, foram utilizadas para permitir o uso de tecnologias semânticas e a recuperação de imagens de máquinas de buscas e serviços de imagens. 


\section{CBIE-LACLO 2015}

Anais dos Workshops do IV Congresso Brasileiro de Informática na Educação (CBIE 2015)

A Figura 2 representa o workflow de criação do jogo. Basicamente, o jogo ISCOOL é dividido em duas partes principais: (i) processamento de texto; e (ii) criação do jogo.

Na primeira parte, (i) processamento de texto, o ISCOOL utiliza uma gama de ferramentas e conjuntos de dados publicados livremente na Web para criar e instanciar elementos básicos do jogo. Como cada jogo do ISCOOL é criado a partir de um texto inserido pelo jogador, o primeiro passo do workflow para criação do jogo é processar o texto inserido pelo jogador. Sendo assim, dado como entrada um texto livre, o ISCOOL irá processar o texto para extrair informações sobre fatos, conceitos, entidades e adicionar definições de palavras que possam auxiliar o jogador a compreender o texto e posteriormente jogar o jogo criado.

O processamento de texto é feito através de ferramentas semânticas como é o caso do DBpedia Spotlight ${ }^{1}$ e Wordnet $^{2}$, enquanto que as definições de palavras são obtidas através da Wikitionary ${ }^{3}$. O DBpedia Spotlight é uma ferramenta de reconhecimento de entidades em texto, isto é, identifica pessoas, organizações, lugares, etc. Por exemplo, na frase "Bill Gates e Paul Allen fundaram a Microsoft.", possivelmente três entidades seriam reconhecidas: as pessoas Bill Gates e Paul Allen e também a empresa Microsoft. Essa ferramenta então cria hyperlinks entre entidades encontradas no texto e suas descrições estruturadas na DBpedia ${ }^{4}$. O Wordnet é uma base de dados léxica na qual substantivos, adjetivos, verbos e advérbios estão agrupados em synsets. Synsets representam grupos de palavras que estão interligados por meio de relações conceituais-semânticas e lexicais. Por fim, o Wikitionary serve como base de dados adicional para fornecer definições de palavras, sinônimos, antônimos, hiperonímias, etc.

Desta forma, a primeira parte (i) é responsável por processar o texto e extrair informações e/ou interligar informações com bases de dados externas para servir como entrada para a próxima etapa e a criação do jogo.

A segunda parte é responsável pela criação do jogo em si, ou seja, obter imagens advindas de máquinas de buscas e outros serviços de imagens como, por exemplo, Flickr ${ }^{5}$ e Bing APIs ${ }^{6}$. As imagens são obtidas a partir das entidades identificadas no texto inserido pelo jogador (por exemplo, o termo "Microsoft"). Além disso, como as entidades estão descritas de forma estruturada na DBpedia, obtemos também informações sobre a categoria a qual uma entidade está classificada (por exemplo, no caso da Microsoft, uma possível categoria seria "Empresas de Informática"). Assim, as categorias associadas a cada entidade no texto servem como entrada para a recuperação de imagens adicionais para criar o jogo (ii). Para cada imagem, recuperada a partir do Bing/Flickr, é designada uma pontuação de relevância.

O ISCOOL é parcialmente baseado na premissa de que, caso o jogador seja capaz de identificar as entidades representadas como imagens no jogo, então algum grau de compreensão sobre o conteúdo ele possuirá. Além disso, a pontuação do jogo segue

\footnotetext{
${ }^{1}$ http://dbpedia-spotlight.github.io/demo/

${ }^{2}$ https: //wordnet.princeton.edu

${ }^{3}$ https://wiktionary.org

${ }^{4}$ http: / / dbpedia.org

${ }^{5}$ https: / / www.flickr.com/services/api/

${ }^{6}$ http: / / www.bing.com/dev/
} 


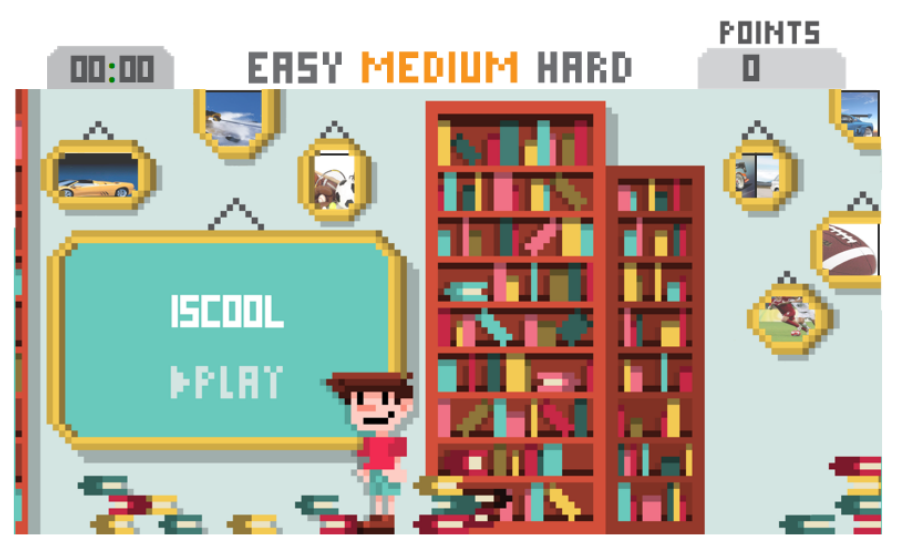

Figura 2. Tela inicial do jogo ISCOOL.

um critério de cobertura, onde quanto mais diversa for a escolha das imagens, ou seja, imagens que representem diferentes entidades/conceitos no texto, maior será a pontuação do jogador.

\section{Apresentação do Software}

Abaixo apresentamos uma breve descrição de como utilizar o jogo ISCOOL através de um possível exemplo de uso em sala de aula. Ressaltamos que este jogo não se limita ao exemplo aqui explicitado e que outras situações pedagógicas, não cobertas nesse artigo, podem ser aplicadas.

Hipoteticamente, imagine que um professor de história criou uma tarefa para seus alunos para ser resolvida utilizando o jogo ISCOOL. Nesta tarefa, o professor extraiu da Wikipedia ${ }^{7}$ um trecho do texto sobre "Revolução Francesa"e solicitou aos seus alunos que identificassem, através do jogo, cinco imagens que melhor refletissem o conteúdo do texto.

Por sua vez, os alunos acessaram o jogo através do link http://ccead. puc-rio.br/iscool/ e inseriram o texto solicitado pelo professor em "input text here" (ver Figura 3). Após inserir o texto, o aluno/jogador deve pressionar o botão "play" para processar o texto e, também, criar o jogo de imagens do ISCOOL de acordo com o texto inserido. A Figura 4 apresenta a saída gerada para o texto inserido pelo jogador com hyperlinks para informações adicionais sobre fatos, conceitos, entidades bem como definições de palavras. Nesta etapa o usuário pode explorar o texto através dos hyperlinks criados para fontes externas antes de começar a jogar. A Figura 5 mostra uma descrição para a entidade "Louis XVI", rei da França, reconhecida no texto de entrada.

Após o texto ser processado, o jogo ISCOOL é criado e disponibilizado para o jogador que deverá selecionar o nível de dificuldade do jogo e só então clicar em "Play" para começar a jogar. O nível de dificuldade está associado ao tempo que o jogador terá para concluir a tarefa e também ao score (pontuação) que este poderá receber (ver Figura 6). De acordo com o nível escolhido, podendo ser fácil, médio ou difícil, o peso dos pontos obtidos é multiplicado por um, dois ou três, respectivamente.

\footnotetext{
${ }^{7}$ https: / / www.wikipedia.org
} 
CBIE-LACLO 2015

Anais dos Workshops do IV Congresso Brasileiro de Informática na Educação (CBIE 2015)

\section{I5ㅁㅁㄴㄴ}

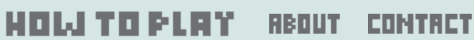

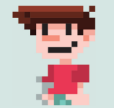

During the reign of Louis XVI, France faced a major economic crisis, partially initiated by the cost of intervening in the American Revolution,

and exacerbated by a regressive system of

taxation. On 5 May 1789 the Estates-General of

1789 convened to deal with this issue, but were

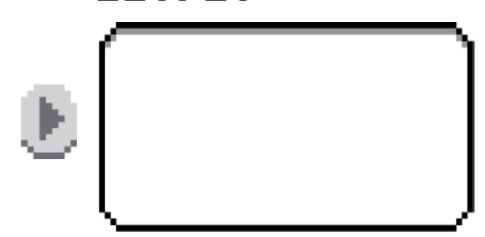

Figura 3. Entrada de dados pelo aluno/jogador.

\section{वUTFUT}

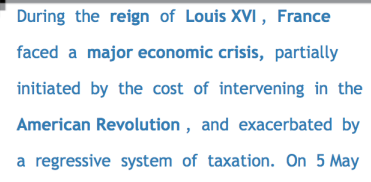

initiated by the cost of intervening in the

American Revolution, and exacerbated by

a regressive system of taxation. On 5 May

Figura 4. Resultado do processamento de texto de entrada.

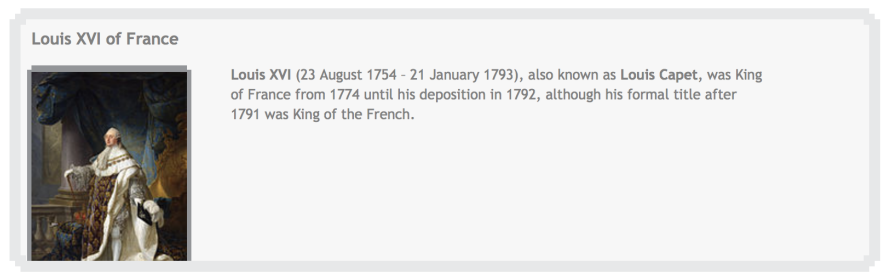

Figura 5. Informação extraída da DBpedia sobre a entidade "Louis XVI".

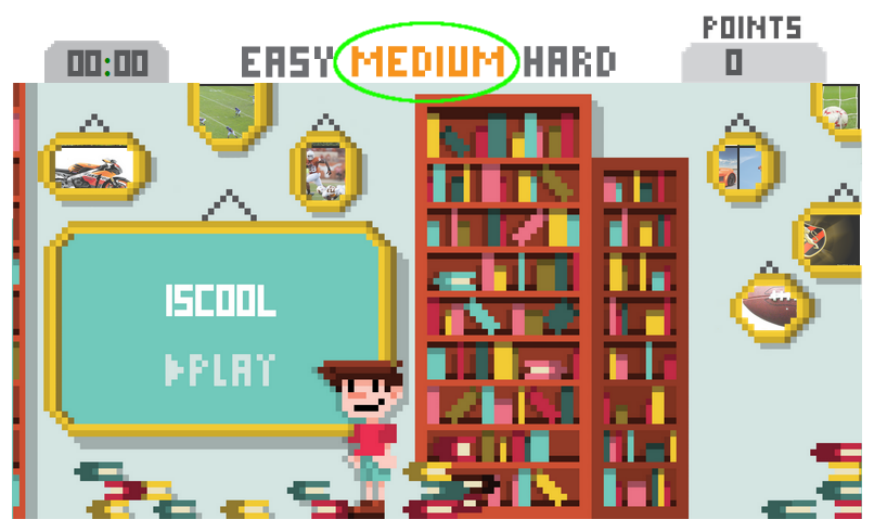

Figura 6. Jogo ISCOOL - tela inicial e seleção de nível de dificuldade.

Ao selecionar o nível de dificuldade médio (medium), um grid com 49 imagens é mostrado ao jogador (ver Figura 7). O grid é montado dinamicamente e durante o decorrer do tempo novas imagens são aleatoriamente inseridas, relacionadas ou não com 


\section{CBIE-LACLO 2015}

Anais dos Workshops do IV Congresso Brasileiro de Informática na Educação (CBIE 2015)

o texto de entrada. O jogador deve ser ágil e atento para selecionar as imagens que melhor representam o texto de entrada.

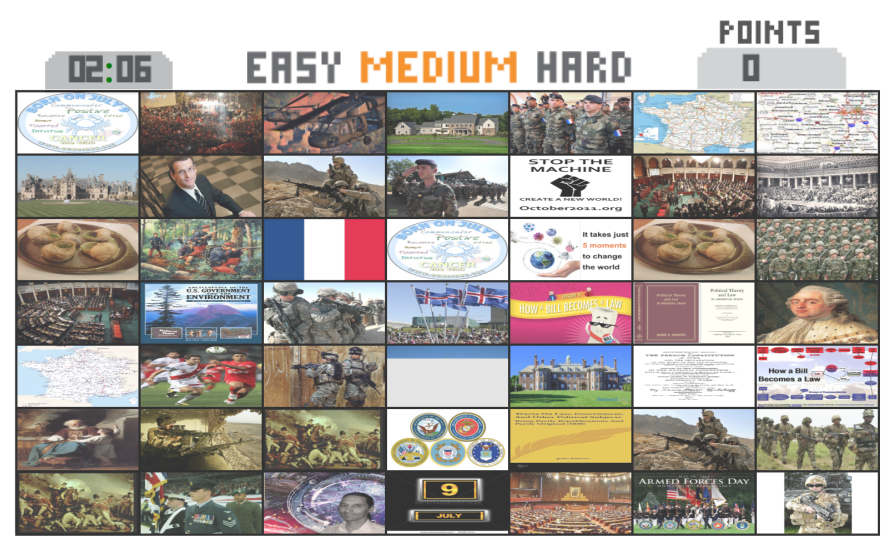

Figura 7. Jogo ISCOOL em ação.

Quando o jogador terminar de selecionar as imagens correspondentes ao texto de entrada, uma tela com a pontuação do jogador e as imagens selecionadas é mostrada (ver Figura 8).

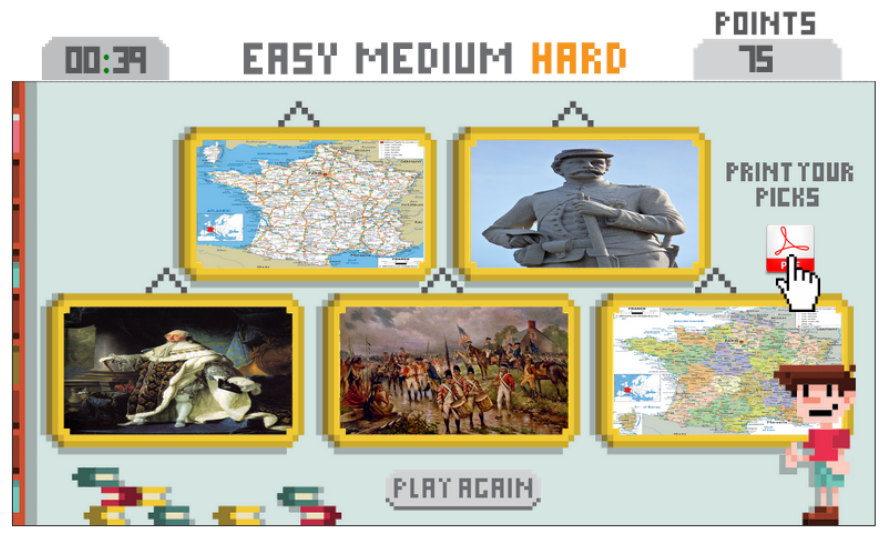

Figura 8. Imagens selecionadas e possibilidade de impressão do resultado.

Por fim, o jogador pode salvar o arquivo gerado em PDF e enviar ao professor que solicitou a tarefa ou jogar novamente.

Para mais informações sobre como jogar, nos referimos a página http:// ccead.puc-rio.br/iscool/how.php.

\section{Trabalhos relacionados}

Recentemente diversas abordagens online têm sido desenvolvidas para suportar a educação a distância, muitas das quais têm sido possíveis devido ao desenvolvimento de tecnologias semânticas e à adaptação de ferramentas existentes para um ambiente de hipertexto dinâmico. Alguns exemplos são discutidos a seguir: (i) a caneta marca-texto tradicional foi reinventada como uma versão online para apoiar os alunos matriculados em cursos online [Nunes et al. 2012], apresentando diversas vantagens sobre o original 


\section{CBIE-LACLO 2015}

Anais dos Workshops do IV Congresso Brasileiro de Informática na Educação (CBIE 2015)

como as possibilidades de compartilhamento entre alunos, ser utilizado para melhorar o conteúdo do curso e servir como uma âncora para conectar-se a novos recursos na Web; (ii) outra ferramenta de e-learning [Nunes et al. 2015b] analisa semanticamente o conteúdo de mensagens em fóruns educacionais, muitos dos quais com centenas de mensagens individuais, fornecendo aos instrutores uma visão geral das discussões pela sumarização do conteúdo de um fórum através de tópicos extraídos da DBpedia; (iii) outra abordagem [Nunes et al. 2014a, Nunes et al. 2014b] utiliza tópicos extraídos de mensagens em um fórum para recomendar outras discussões que possam enriquecer a atual. O ISCOOL usa métodos semelhantes para recuperar imagens através de motores de busca visando ajudar e apoiar os alunos na interpretação e análise de textos.

Além disso, jogos sérios começaram a emergir como abordagens complementares ou alternativas para atividades de aprendizagem tradicionais. A combinação de entretenimento e atividades educativas envolve os alunos, incentivando-os a atingir as metas de aprendizagem, consequentemente impactando em seu desempenho acadêmico de uma maneira positiva [Derbali and Frasson 2012]. Jogos sérios têm sido capazes de cativar e envolver os alunos para propósitos específicos tais como o desenvolvimento de novos conhecimentos ou a aquisição de conjuntos de habilidades específicas. Kurkovsky [Kurkovsky 2009] mostra que os jogos também podem contribuir para melhorar as taxas de retenção em disciplinas difíceis, quando usados para complementar o material do curso [Morrison and Preston 2009]. Seguindo essa tendência, o ISCOOL utiliza a abordagem de jogos sérios aplicada ao contexto educacional.

\section{Considerações Finais}

Imagens desempenham um papel extremamente importante nos processos de aprendizagem e interpretação de texto em qualquer nível de ensino. Isto é particularmente verdadeiro no caso dos dois grupos demográficos identificados que podem se beneficiar diretamente do jogo ISCOOL: pessoas com diferentes níveis de deficiência auditiva e pessoas com níveis limitados de alfabetização. Em ambos os casos, recursos visuais são fundamentais como uma ferramenta para derivar significados de palavras, conceitos e textos.

Testes preliminares realizados com a ajuda de pessoas com baixo nível de alfabetização foram bem sucedidos. Atualmente está em curso a segunda fase de testes e avaliação do jogo ISCOOL com pessoas com deficiência auditiva.

Além disso, o ISCOOL permite a capacitação e o enriquecimento na vida daqueles que são menos favorecidos através do uso básico de tecnologias semânticas aliadas a jogos sérios. O uso de tecnologias semânticas e de recuperação de imagens da Web permite que o jogo se adapte de forma a contemplar diferentes conteúdos e atividades que possam melhorar o processo de ensino-aprendizagem.

Como trabalho futuro pretende-se adicionar ao ISCOOL a possibilidade de criar grupos de jogadores para resolver tarefas específicas e permitir que o criador da tarefa possa acompanhar o desenvolvimento dos seus jogadores. Além disso, pretende-se criar um processo semi-automático de geração de tarefa. Atualmente, cada jogador deve introduzir um texto e o jogo é gerado automaticamente, contudo prevemos a criação de um processo semi-automático onde o criador da tarefa poderá pré-selecionar imagens, corretas ou não.

Por fim, maiores detalhes técnico/científicos sobre o ISCOOL podem ser obti- 


\section{CBIE-LACLO 2015}

Anais dos Workshops do IV Congresso Brasileiro de Informática na Educação (CBIE 2015)

dos em [Nunes et al. 2015a]. A ferramenta está disponível em http://research. ccead.puc-rio.br/iscool.

\section{Agradecimentos}

Este trabalho foi parcialmente financiado pela FAPERJ (processo no. E26/190.061/2014) e pelo CNPq (processo no. 444976/2014-0).

\section{Referências}

Derbali, L. and Frasson, C. (2012). Assessment of learners' motivation during interactions with serious games: A study of some motivational strategies in food-force. Adv. in Hum.-Comp. Int., 2012:5:5-5:5.

Kurkovsky, S. (2009). Engaging students through mobile game development. In Proceedings of the 40th ACM Technical Symposium on Computer Science Education, SIGCSE '09, pages 44-48, New York, NY, USA. ACM.

Morrison, B. B. and Preston, J. A. (2009). Engagement: Gaming throughout the curriculum. In Proceedings of the 40th ACM Technical Symposium on Computer Science Education, SIGCSE '09, pages 342-346, New York, NY, USA. ACM.

Nunes, B. P., Caraballo, A. A. M., Kawase, R., Fetahu, B., Casanova, M. A., and de Campos, G. H. B. (2014a). A topic extraction process for online forums. In IEEE 14th International Conference on Advanced Learning Technologies, ICALT 2014, Athens, Greece, July 7-10, 2014, pages 541-543. IEEE.

Nunes, B. P., Kawase, R., Dietze, S., de Campos, G. H. B., and Nejdl, W. (2012). Annotation tool for enhancing e-learning courses. In Popescu, E., Li, Q., Klamma, R., Leung, H., and Specht, M., editors, Advances in Web-Based Learning - ICWL 2012 11th International Conference, Sinaia, Romania, September 2-4, 2012. Proceedings, volume 7558 of Lecture Notes in Computer Science, pages 51-60. Springer.

Nunes, B. P., Kawase, R., Fetahu, B., Casanova, M. A., and de Campos, G. H. B. (2014b). Educational forums at a glance: Topic extraction and selection. In Benatallah, B., Bestavros, A., Manolopoulos, Y., Vakali, A., and Zhang, Y., editors, Web Information Systems Engineering - WISE 2014 - 15th International Conference, Thessaloniki, Greece, October 12-14, 2014, Proceedings, Part II, volume 8787 of Lecture Notes in Computer Science, pages 351-364. Springer.

Nunes, B. P., Nurmikko-Fuller, T., Lopes, G. R., and Renso, C. (2015a). A serious game powered by semantic web technologies. In Proceedings of the 24th International Conference on World Wide Web Companion, WWW'15 Companion, pages 227-230, Republic and Canton of Geneva, Switzerland. International World Wide Web Conferences Steering Committee.

Nunes, B. P., Tyler-Jones, M., de Campos, G. H. B., Siqueira, S. W. M., and Casanova, M. A. (2015b). Fat: A real-time (f)orum (a)ssessment (t)ool to assist tutors with discussion forums assessment. In Proceedings of the 30th Annual ACM Symposium on Applied Computing, SAC '15, New York, NY, USA. ACM. 